\title{
ON THE QUALITATIVE ANALYSIS OF SOME STATIC CYLINDRICALLY SYMMETRIC SOLUTIONS OF THE EINSTEIN EQUATIONS
}

\author{
S. B. Grigoryev, A. B. Leonov \\ Oles Honchar Dnipropetrovsk National University \\ 72, Gagarina Ave., Dnipropetrovsk, UA-49010, Ukraine \\ e-mail: arkadiyleonov@yahoo.com
}

(Received by July 7, 2015; in final form - April 12, 2016)

\begin{abstract}
We perform a qualitative analysis of the cylindrically symmetric static Einstein equations with the diagonal stress-energy tensor with mutually proportional components. This case simultaneously covers several important exact solutions, such as the solution with the static electric field, the perfect fluid a solution, solution with the cosmological constant and the massless scalar field solution. The problem involves the analysis of the system in a neighborhood of a non-hyperbolic equilibrium point. However, due to the properties of the system it is possible to study it qualitatively in the whole phase plane.
\end{abstract}

Key words: Einstein equations, cylindrical symmetry, dynamical systems.

PACS number(s): 04.20.Jb

\section{INTRODUCTION}

We continue our study of the Einstein equations in the static cylindrically symmetric case in the presence of the stress-energy tensor of the form (1)

$$
T_{\nu}^{\mu}=\left(\begin{array}{cccc}
l p & 0 & 0 & 0 \\
0 & -p & 0 & 0 \\
0 & 0 & -m p & 0 \\
0 & 0 & 0 & -n p
\end{array}\right)
$$

where $p$ is the pressure and $l, m$ and $n$ are constants. The stress-energy tensor of this form encompasses several important cases (see Table), such as the case of the static electric field, the massless scalar field, the cosmological constant and the perfect fluid. Thus, it is possible to study all these solutions simultaneously.

\begin{tabular}{|l|c|c|c|c|}
\hline & $l$ & $m$ & $n$ & $k$ \\
\hline Static electric field & -1 & -1 & -1 & -1 \\
\hline Massless scalar field & 1 & -1 & -1 & 0 \\
\hline Cosmological constant & -1 & 1 & 1 & 1 \\
\hline Stationary perfect fluid & $\alpha$ & 1 & 1 & $\frac{3+\alpha}{2}$ \\
\hline
\end{tabular}

Table. Various cases covered by the stress-energy tensor of the form (1). The constant $k$ arises in the process of solving the Einstein equations and is defined as $k=\frac{1}{2}(l+m+n+1)$.

In our first paper on the topic [1] we showed that, depending on the values of the parameters $l, m$ and $n$, the system of Einstein equations can be reduced to the system of two ordinary differential equations. We also integrated the system for the case $n=-1, k \neq 0$.

In the present paper we study this system qualitatively. Due to the quadratic nature of its equations, we were able to derive the general properties of its phase portraits, valid in the whole phase plane and derive the exact expression for its integral curves. Every set of parameters $l, m$ and $n$, in fact, encodes a particular type of matter content in the gravitating system - the methods of the theory of dynamical systems allow to perform a classification of the solutions (and corresponding stress-energy tensors) relatively to the qualitative behavior of the integral curves of the said solutions. In $[2,3]$ the same type of analysis was applied to the case of spherical symmetry.

The exact cylindrically symmetric solutions have been extensively studied. The vacuum and the most important non-vacuum solutions can be found in [6]. For the study of solutions with the electromagnetic field see [7-9]. The solution with the static electric field had been obtained in [11], and the values of the parameters $l=-1, m=-1$, $n=-1$ allow it to be described by tensor (1).

The static cylindrically symmetric perfect fluid solution has been studied under different assumptions in [13-16]. The exact perfect fluid solution with the equation of state $\epsilon=\alpha p$ has been found in [19]. The discussion of the stationary rotating fluid can be found in $[17,18]$. The cylindrically symmetric solution with the cosmological constant can be found in [20]. Cylindrical symmetry provides a simple model for the study of gravitomagnetic effects in astrophysics - see [21]. The solution with the nonlinear conformally invariant scalar field has been studied in [12].

The methods of the theory of dynamic systems have found a noticeable application in cosmology: see general overview in [22] and examples of the applications to particular models in [23,24], as well as in [25] and [26]. For an example of the application to exact solutions see [27].

\section{SYSTEM OF EQUATIONS}

Studying the cylindrically symmetric static case we start with the following ansatz for the metric tensor:

$$
d s^{2}=e^{2 U} d t^{2}-e^{2 K-2 U}\left(d \rho^{2}+d z^{2}\right)-W^{2} e^{-2 U} d \varphi^{2}
$$


where $\rho$ is the radial coordinate, and the coordinate $z$ runs along the axis of symmetry. The functions $U, K$ and $W$ depend only on $\rho$.

Then, the Einstein equations take the form

$$
\begin{aligned}
& e^{2 U-2 K}\left(2 U^{\prime \prime}+2 U^{\prime} \frac{W^{\prime}}{W}-U^{\prime 2}-K^{\prime \prime}-\frac{W^{\prime \prime}}{W}\right)=8 \pi l p \\
& e^{2 U-2 K}\left(U^{\prime 2}-K^{\prime} \frac{W^{\prime}}{W}\right)=-8 \pi p \\
& -e^{2 U-2 K}\left(K^{\prime \prime}+U^{\prime 2}\right)=-8 \pi m p \\
& -e^{2 U-2 K}\left(U^{\prime 2}-K^{\prime} \frac{W^{\prime}}{W}+\frac{W^{\prime \prime}}{W}\right)=-8 \pi n p
\end{aligned}
$$

The system consists of the equations of the second order, but one can notice that, in fact, only the first and second derivatives enter the system: for $U, K$ one can see it right away, and the terms with $W$ boil down to the derivatives of $\ln W$. Thus, we are dealing with a first order system.

Then, as presented in (1), this system can be integrated once with the help of the following transformation

$$
\begin{aligned}
U^{\prime} & =k F \\
K^{\prime} & =(m+1) F-C_{H} H \\
\frac{W^{\prime}}{W} & =(n+1) F+H,
\end{aligned}
$$

where $C_{H}$ is the constant that arises as the result of the integration.

In the end, it transforms the initial system into

$$
\begin{aligned}
F^{\prime} & =\left(m(n+1)-k^{2}\right) F^{2} \\
& +\left(m-(n+1) C_{H}\right) F H-C_{H} H^{2} \\
H^{\prime} & =-(n+1) F H-H^{2} .
\end{aligned}
$$

This transformation is motivated foremost by the mathematical considerations, as the transformed system makes the qualitative analysis easier and more transparent. Note though that the transformation imposes restrictions on the possible physical cases. The transformation is valid only if $k \neq 0$. Thus, the transformed system describes the case of the static electric field, the perfect fluid case and the cosmological constant case, but the case with the massless scalar field is not described.

In order to make some progress with this system we are going to use the following transformation to the new functions $R(\rho)$ and $\phi(\rho)$

$$
\begin{aligned}
& F=R \cos \phi \\
& H=R \sin \phi .
\end{aligned}
$$

Our goal is to study the system (10)-(11) qualitatively, so we are going to sketch phase portraits of the system. On the phase portrait of the system (10)-(11) functions $F$ and $H$ play the role of the Cartesian coordinates, thus this is just a transformation to polar coordinates on a phase portrait.

The transformation is useful because the right-hand parts of the system (10)-(11) contain only second degree terms. Inserting (12)-(13) into the right-hand parts of (10)-(11) we obtain

$$
\begin{aligned}
F^{\prime} & =R^{2}\left[\left(m(n+1)-k^{2}\right) \cos ^{2} \phi\right. \\
& \left.+\left(m-(n+1) C_{H}\right) \cos \phi \sin \phi-C_{H} \sin ^{2} \phi\right] \\
H^{\prime} & =R^{2}\left[-(n+1) \cos \phi \sin \phi-\sin ^{2} \phi\right] .
\end{aligned}
$$

As we can see, the right-hand sides break into parts that depend separately on just $R$ and $\phi$.

Now, the derivatives of $F$ and $H$ are

$$
\begin{aligned}
& F^{\prime}=R^{\prime} \cos \phi-R \phi^{\prime} \sin \phi \\
& H^{\prime}=R^{\prime} \sin \phi+R \phi^{\prime} \cos \phi,
\end{aligned}
$$

so, system (10)-(11) after transformation (12)-(13) yields

$$
\begin{aligned}
& R^{\prime} \cos \phi-R \phi^{\prime} \sin \phi=R^{2}\left[\left(m(n+1)-k^{2}\right) \cos ^{2} \phi+\left(m-(n+1) C_{H}\right) \cos \phi \sin \phi-C_{H} \sin ^{2} \phi\right] \\
& R^{\prime} \sin \phi+R \phi^{\prime} \cos \phi=R^{2}\left[-(n+1) \cos \phi \sin \phi-\sin ^{2} \phi\right] .
\end{aligned}
$$

Multiplying by the sines and cosines and reshuffling the equations, we extract the derivatives $R^{\prime}$ and $\phi^{\prime}$, and arrive at the system of equations of our main interest:

$$
\begin{aligned}
& R^{\prime}=R^{2}\left[\left(m(n+1)-k^{2}\right) \cos ^{3} \phi+\left(m-(n+1) C_{H}\right) \cos ^{2} \phi \sin \phi-\left(n+1+C_{H}\right) \cos \phi \sin ^{2} \phi-\sin ^{3} \phi\right] \\
& R \phi^{\prime}=R^{2} \sin \phi\left[\left(k^{2}-(m+1)(n+1)\right) \cos ^{2} \phi-\left(m+1-(n+1) C_{H}\right) \cos \phi \sin \phi+C_{H} \sin ^{2} \phi\right] .
\end{aligned}
$$

We also introduce the following notation

$$
\begin{aligned}
& P(\phi)=\left(m(n+1)-k^{2}\right) \cos ^{3} \phi+\left(m-(n+1) C_{H}\right) \cos ^{2} \phi \sin \phi-\left(n+1+C_{H}\right) \cos \phi \sin ^{2} \phi-\sin ^{3} \phi . \\
& Q(\phi)=\sin \phi\left[\left(k^{2}-(m+1)(n+1)\right) \cos ^{2} \phi-\left(m+1-(n+1) C_{H}\right) \cos \phi \sin \phi+C_{H} \sin ^{2} \phi\right] .
\end{aligned}
$$


Immediately we see that we can integrate (20)-(21)

$$
\int_{R_{0}}^{R} \frac{d R}{R}=\int_{\phi_{0}}^{\phi} d \phi \frac{P(\phi)}{Q(\phi)} .
$$

This integral can be evaluated explicitly, which we will do in the final chapter of this paper.

It proves more useful to study the system of equations (20)-(21) qualitatively. Our goal is to built the phase portrait of this system in the neighborhood of its equilibrium points - it gives us most of the information about the behavior of its solutions, and allows us to study the dependence of the overall behavior of the system on the parameters $k, m, n$ and $C_{H}$.

\section{QUALITATIVE ANALYSIS}

\section{A. Separatrices and Sectors}

In order to qualitatively study the system of equations one has to determine its equilibrium points. The equilibrium points of the system (10)-(11) (and as a consequence, the system $(20)-(21))$ were found in our preceding paper [1].

This system has one non-hyperbolic equilibrium point at the origin $R=0$. However, if the values of the parameters $k, m, n$ satisfy the condition $k^{2}=m(n+1)$, the system acquires a set of equilibrium points on the line $\sin \phi=0$. Thus, the systems that describe the static electric field, the perfect fluid and the cosmological constant have only a non-hyperbolic equilibrium point at $R=0$.

To determine the type of the equilibrium point, we have to study, at first, the linear terms of the Taylor expansion of the system. However, the system is purely quadratic, so the linear terms are all zero in the neighborhood of the point $R=0$, thus this point is not hyperbolic. The general method for studying the non-hyperbolic equilibrium points can be found in $[4,5]$. We use a less general method, but more suitable for our particular system.

First of all, let us examine those points of phase plane where the derivative $\phi^{\prime}$ turns to zero.

$$
\begin{aligned}
\phi^{\prime} & =R^{2} \sin \phi\left[\left(k^{2}-(m+1)(n+1)\right) \cos ^{2} \phi\right. \\
& \left.-\left(m+1-(n+1) C_{H}\right) \cos \phi \sin \phi+C_{H} \sin ^{2} \phi\right]=0 .
\end{aligned}
$$

In general, such equation should implicitly define some intricate line. But in our case the dependence of $R$ and $\phi$ is neatly separated, so we get only an equation in $\phi$.

$$
\begin{aligned}
\sin \phi & {\left[\left(k^{2}-(m+1)(n+1)\right) \cos ^{2} \phi\right.} \\
& \left.-\left(m+1-(n+1) C_{H}\right) \cos \phi \sin \phi+C_{H} \sin ^{2} \phi\right]=0 .
\end{aligned}
$$

This means that for every solution of this equation (let us denote them as $\phi_{k}^{R}$ ) we get a ray, starting at the equilibrium point $R=0$, and in every point of this ray we have $\phi^{\prime}=0$. But it means that the vector field, defined by our system on the phase plane, is tangent to these rays. Thus, each ray $\phi=\phi_{k}^{R}$ is a solution of the system (20)-(21).

Other solutions cannot cross these rays, thus rays break the phase plane into a number of sectors. Any solution, starting in a particular sector, always stays inside this sector. And this property holds on the whole phase plane, as we are not performing any series expansions near the equilibrium point, but use the original equations of the system.

In our case, equation $\phi^{\prime}=0$ always has some roots, so the system does not have periodic solutions. We will call solutions $\phi=\phi_{k}^{R}$ of equation (26) separatrices. As we will see, they break the phase plane into parts with the different qualitative behavior of the solutions, and largely determine this behavior. A part of the phase plane between two separatrices $\phi_{1}^{R}$ and $\phi_{2}^{R}\left(\phi_{2}^{R}>\phi_{1}^{R}\right)$ with no other separatrices between them we will call a sector.

\section{B. Qualitative Properties of the Integral Curves}

Let us get back to integral (24). It gives us solutions in the form of the integral curves $R=R(\phi)$. We want to study the qualitative behavior of these curves inside sectors defined by the separatrices.

Suppose that we have a sector defined by two separatrices $\phi_{1}^{R}$ and $\phi_{2}^{R}\left(\phi_{2}^{R}>\phi_{1}^{R}\right)$; and two points $\left(R_{1}, \phi_{1}\right)$ and $\left(R_{2}, \phi_{2}\right)$, in between these separatrices: $\phi_{2}^{R}>\phi_{2}>\phi_{1}>$ $\phi_{1}^{R}$. Then integral (24) evaluated between these points is finite. Let us perform the substitution $v=\cot \phi$ (together with the appropriate change of limits of integration $\left.v_{1}=\cot \phi_{1}, v_{2}=\cot \phi_{2}\right)$. First of all we introduce the following notation

$$
\begin{aligned}
\bar{P}(v) & =\left(m(n+1)-k^{2}\right) v^{3}+\left(m-(n+1) C_{H}\right) v^{2} \\
& -\left(n+1+C_{H}\right) v-1 \\
\bar{Q}(v) & =\left(k^{2}-(m+1)(n+1)\right) v^{2} \\
& -\left(m+1-(n+1) C_{H}\right) v+C_{H} .
\end{aligned}
$$

And after the substitution we get

$$
\int_{R_{1}}^{R_{2}} \frac{d R}{R}=-\int_{v_{1}}^{v_{2}} d v \frac{1}{\left(1+v^{2}\right)} \cdot \frac{\bar{P}(v)}{\bar{Q}(v)} .
$$

These integral can be divergent under two conditions. First: if the denominator turns to zero. But the denominator, in fact, is just equation (26) that defines the separatrices. And we assumed that the limits of integration lie between two neighboring separatrices. Second: we used the substitution $v=\cot \phi$ which is not valid for points $\sin \phi=0$. However, looking at equation (26), we see that the points $\sin \phi=0$ are separatrices themselves, thus this condition is also not triggered. It follows then that the function $R=R(\phi)$ is a single-valued, bounded function on every domain $\left(\phi_{1}, \phi_{2}\right)$ satisfying $\phi_{2}^{R}>\phi_{2}>\phi_{1}>\phi_{1}^{R}$. 


\section{S. B. GRIGORYEV, A. B. LEONOV}

This property greatly simplifies the study of our system. It is typical only for the homogeneous systems of two equations, though.

In order to sketch a phase portrait of our system we are mainly interested in the information about the behavior of the function $R=R(\phi)$ near the separatrices. We can determine this behavior studying the inclination of the graph of $R=R(\phi)$ near the separatrices, or, in other words, studying the sign of $\frac{d R}{d \phi}$.

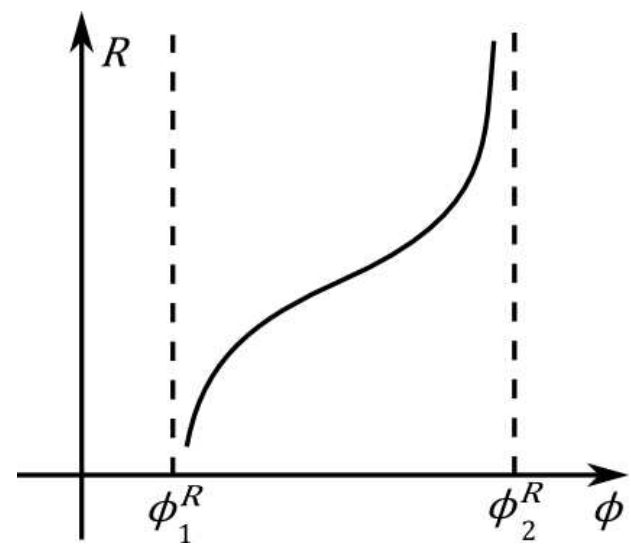

Fig. 1. An integral curve $R=R(\phi)$ plotted as a graph in the Cartesian coordinates. Dashed lines are separatrices situated at the angles $\phi_{1}^{R}$ and $\phi_{2}^{R}$.

Suppose we are given the integral curve $R=R(\phi)$ inside some particular sector on the phase plane. Let us draw its graph in the Cartesian coordinates (see figure 1). Now, from (20)-(21) we have

$$
\frac{d R}{d \phi}=\frac{R P(\phi)}{Q(\phi)}
$$

First of all, we are interested in the number of extrema of the $R=R(\phi)$. From the numerator of the above expression we see that in the range $0<\phi<2 \pi$ the number of zeros of the $\frac{d R}{d \phi}$ is finite, thus the number of extrema is also finite. It means that every separatrice has a neighborhood where the function $R=R(\phi)$ is monotonic. So, in the neighborhood of a separatrice the function $R=R(\phi)$ always tends to either 0 or infinity, and the sign of $\frac{d R}{d \phi}$ corresponds to these directions.

But how should we find the sign of $\frac{d R}{d \phi}$ ? Let us not forget that (see (20)-(21)):

$$
\frac{d R}{d \phi}=\frac{R R^{\prime}(\phi)}{\phi^{\prime}(\phi)} .
$$

The vector field, defined by the system of equations on the phase space, is at least once continuously differentiable, so in the small neighborhood of some separatrice $\phi^{R}$ the sign of $R^{\prime}(\phi)$ is the same as the sign of the derivative $R^{\prime}\left(\phi^{R}\right)$ on the separatrice. The sign of $R^{\prime}\left(\phi^{R}\right)$ can be directly found using (20). We know $\phi^{R}$, simply insert it into $(20)$ and calculate the sign. $\phi^{\prime}(\phi)$ is zero on every separatrice, which means that the sign of $\phi^{\prime}$ is constant across any given sector. Thus we can calculate the sign of $\phi^{\prime}(\phi)$ in any sector from equation (21) (also see figure $(2))$.

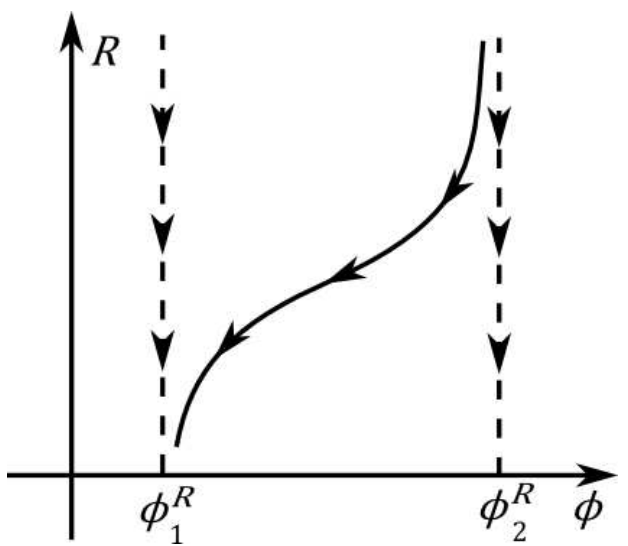

Fig. 2. The direction of the separatrices $\phi_{1}^{R}$ and $\phi_{2}^{R}$ and the sign of $\phi^{\prime}(\phi)$ inside the sector determine the asymptotic behavior of the integral curve $R=R(\phi)$ and its direction. Here $R^{\prime}\left(\phi_{1}^{R}\right), R^{\prime}\left(\phi_{2}^{R}\right)$ and $\phi^{\prime}(\phi)$ are all negative.

\section{Summary of the Qualitative Analysis}

Let us make a little summary of the qualitative analysis of the system (20)-(21). To sketch the phase portrait of this system one has to go through the following steps.

1. Solve equation (26) and find the separatrices $\phi_{k}^{R}$ :

$$
\begin{aligned}
& \sin \phi\left[\left(k^{2}-(m+1)(n+1)\right) \cos ^{2} \phi\right. \\
& \left.-\left(m+1-(n+1) C_{H}\right) \cos \phi \sin \phi+C_{H} \sin ^{2} \phi\right]=0 .
\end{aligned}
$$

2. Find the direction of the separatrices. To do it, find the signs of $R^{\prime}(\phi)$ on the separatrices inserting $\phi_{k}^{R}$ into equation (20). The plus sign corresponds to the direction from the equilibrium point $R=0$ to the infinity, the minus sign is a reverse direction.

$$
\begin{aligned}
R^{\prime}= & R^{2}\left[\left(m(n+1)-k^{2}\right) \cos ^{3} \phi\right. \\
& +\left(m-(n+1) C_{H}\right) \cos ^{2} \phi \sin \phi \\
& \left.-\left(n+1+C_{H}\right) \cos \phi \sin ^{2} \phi-\sin ^{3} \phi\right] .
\end{aligned}
$$

3. Find the domains of the positive and negative values of $\phi^{\prime}$ as a function of angle $\phi$ on the phase plane, using equation (26).

$$
\begin{aligned}
& \phi^{\prime}(\phi)=\sin \phi\left[\left(k^{2}-(m+1)(n+1)\right) \cos ^{2} \phi\right. \\
& \left.-\left(m+1-(n+1) C_{H}\right) \cos \phi \sin \phi+C_{H} \sin ^{2} \phi\right] .
\end{aligned}
$$

4. Find the inclination and, as a consequence, the limits (zero or infinity) of the integral curves in the neghborhood of the separatrices. Use the information about the sign of $R^{\prime}(\phi)$ and $\phi^{\prime}(\phi)$ to find the $\operatorname{sign}$ of $\frac{d R}{d \phi}$.

$$
\frac{d R}{d \phi}=\frac{R R^{\prime}(\phi)}{\phi^{\prime}(\phi)}
$$


5. As one can see from (21) all the separatrices apart from $\phi^{R}=0$ and $\phi^{R}=\pi$ will be expressed through the cotangents. The cotangent has a period of $\pi$, so all separatrices will come in pairs of rays, which lie on the same line. Thus we may vary $\phi$ only from 0 to $\pi$, and look only for the separatrices located in the upper half of the phase plane. For every separatrice in the upper half-plane there is a corresponding separatrice in the lower half-plane, lying on the same line. Carrying $\sin \phi$ out of the brackets in $(20)$, we see that the direction of the separatrice in the lower half-plane is the opposite of the corresponding separatrice in the upper half-plane. That is, if the separatrice in the upper half-plane is directed to the infinity, then the separatrice in the lower half-plane is directed towards the equilibrium point.

\section{STATIC ELECTRIC FIELD AS AN EXAMPLE OF ANALYSIS}

As an example, we are going to study the system with static electric fields. The values of the stress-energy tensor parameters for this case are $l=-1, m=-1, n=-1$, $k=-1$. The constant $C_{H}$ is related to the distribution of the charge and mass along the axis of symmetry. We will also see how phase portraits depend on its value.

Inserting the values of the parameters into (20)-(21) we have

$$
\begin{aligned}
R^{\prime} & =R^{2}\left(-\cos ^{3} \phi-\cos ^{2} \phi \sin \phi\right. \\
& \left.-C_{H} \cos \phi \sin ^{2} \phi-\sin ^{3} \phi\right) \\
R \phi^{\prime} & =R^{2} \sin \phi\left(\cos ^{2} \phi+C_{H} \sin ^{2} \phi\right) .
\end{aligned}
$$

First of all, we have to find all the separatrices, so we have to solve equation (26).

$$
\sin \phi\left(\cos ^{2} \phi+C_{H} \sin ^{2} \phi\right)=0 .
$$

We immediately see that the number of zeros depends on the sign of $C_{H}$. Let us suppose for now that $C_{H}$ is negative. Then we have

$$
\cot \phi_{ \pm}^{R}= \pm \sqrt{-C_{H}}
$$

And we get two separatrices from $\sin \phi=0$

$$
\begin{aligned}
& \phi_{0}^{R}=0 \\
& \phi_{\pi}^{R}=\pi .
\end{aligned}
$$

As we have said earlier, it is enough to find separatrices only in the upper half-plane. The corresponding separatrices in the lower half-plane can be found using symmetry considerations. All in all, the current system has six separatrices.
To determine the direction of the separatrices we insert the above solutions into (20). For both separatrices $\cot \phi_{ \pm}^{R}$ we get

$$
R_{ \pm}^{\prime}=R_{ \pm}^{2}\left(C_{H}-1\right)
$$

Thus, both separatrices in the upper half-plane are directed towards the equilibrium point.

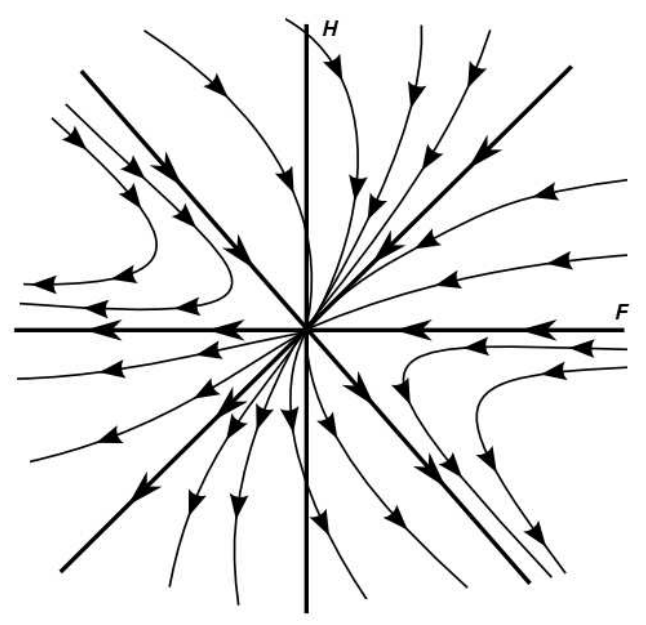

Fig. 3. Phase portrait of the system (10)-(11) sketched using the results of the qualitative analysis. The values of the parameters are $l=-1, m=-1, n=-1, k=-1, C_{H}<0$. The bold straight arrowed lines are separatrices. Arrows represent the directions of integral curves.

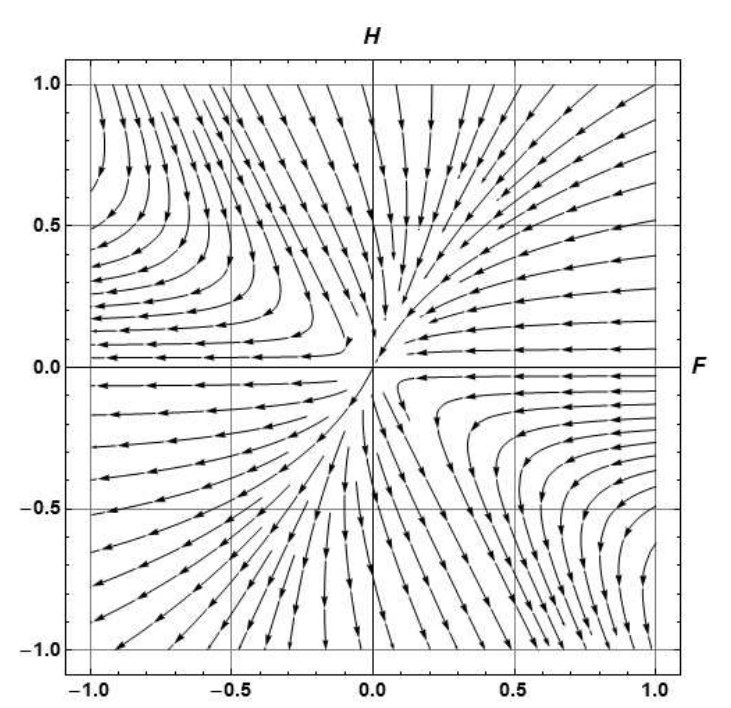

Fig. 4. Numerically created phase portrait of the system (10)-(11). The values of the parameters are $l=-1, m=-1$, $n=-1, k=-1$ and $C_{H}=-1 / 4$.

We also assumed $C_{H}$ to be negative, thus their directions are the same for all permitted values of $C_{H}$.

$$
\begin{aligned}
& R_{0}^{\prime}=-R_{0}^{2} \\
& R_{\pi}^{\prime}=R_{\pi}^{2}
\end{aligned}
$$


To determine the sign of $\phi^{\prime}$ in every sector we again use equation (21), rewriting in our case as

$$
\phi^{\prime}=\sin ^{3} \phi\left(\cot \phi-\cot \phi_{+}^{R}\right)\left(\cot \phi-\cot \phi_{-}^{R}\right) .
$$

Thus, we see that in the first sector (counting counterclockwise from $\phi=0) \phi^{\prime}$ is positive, and then the sign changes in every consecutive sector.

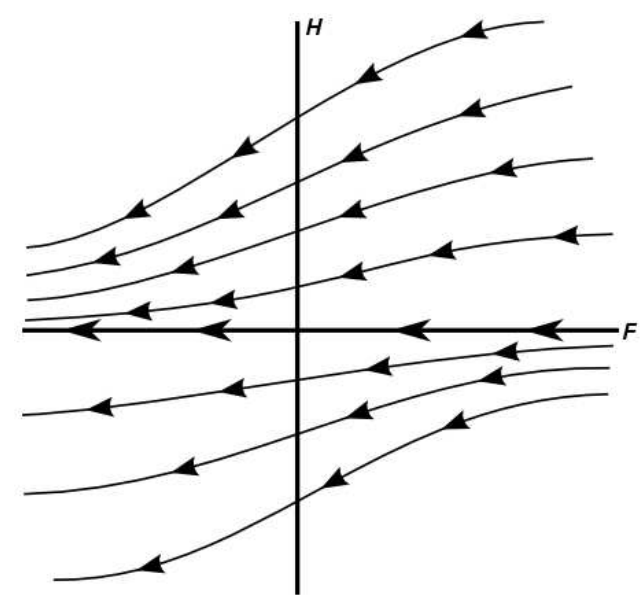

Fig. 5. Phase portrait of the system (10)-(11) with the same values of the parameters as in the previous example: $l=-1, m=-1, n=-1, k=-1$, but with $C_{H}>0$.

Now we can sketch the phase portrait. Combining the information about the signs of $R^{\prime}$ and $\phi^{\prime}$ in different sectors, we obtain Figure 3. It is interesting to compare our qualitative results with a numerically built phase portrait. The latter is presented on Figure 4. Let us get back to equation (38) and sketch the phase portrait for positive $C_{H}$. In this case there are only two separatrices: $\phi_{0}^{R}=0$ and $\phi_{\pi}^{R}=\pi$. Their directions, of course, stay the same, and $\phi^{\prime}$ is positive. The phase portrait is shown on Figure 5 .

\section{PHYSICAL INTERPRETATION}

The system of equations (10)-(11) can have both physical and unphysical solutions. We can use phase portraits to identify the physical solutions, or, at least, the most important of them. We are studying a cylindrically symmetric system with varied matter content, so we are most interested in the cases with sources concentrated on the axis of symmetry - thus all the fields should fall off as we approach infinity. In the case of cylindrical symmetry, however, only forces tend to zero away from the axis, while potentials grow infinitely.

The gravitational forces are described by the Christoffel symbols, and functions $F$ and $H$ are directly related to them.

$$
\begin{aligned}
& \Gamma_{10}^{0}=\Gamma_{01}^{0}=k F ; \\
& \Gamma_{00}^{1}=k F e^{4 U-2 K} ; \\
& \Gamma_{11}^{1}=(m-k+1) F+C_{H} H ; \\
& \Gamma_{22}^{1}=((n+k+1) F-H) W^{2} e^{-2 K} ; \\
& \Gamma_{33}^{1}=-(m-k+1) F-C_{H} H ; \\
& \Gamma_{12}^{2}=\Gamma_{21}^{2}=-(n+k+1) F+H ; \\
& \Gamma_{13}^{3}=\Gamma_{31}^{3}=(m-k+1) F+C_{H} H .
\end{aligned}
$$

All other Christoffel symbols are zero.

We see that Christoffel symbols turn to zero if $F$ and $H$ turn to zero. Now, we need Christoffel symbols to be zero at infinity. In terms of our phase portrait language it means that we are interested in the solutions that approach the coordinate origin on the phase portrait of the system (10)-(11). Our qualitative analysis immediately supplies us with such information.

As an example, let us look at the phase portrait of the system for the case of the electric field, Figure 3). We see that separatrices divide the phase portrait into six parts, and only two of these parts contain solutions that approach the equilibrium point at the origin. Solutions in other parts are either unphysical or correspond to a different distribution of sources.

\section{EXPLICIT EXPRESSION FOR THE INTEGRAL CURVES}

Integral (29) is a rational fraction, so it can be relatively easy integrated to obtain an explicit formula for the integral curves of the system. The only complication arises from the hassle with all the coefficients.

Let us move to indefinite integral in (29) and introduce the following notation

$$
\int \frac{d R}{R}=-\int d v \frac{a_{3} v^{3}+a_{2} v^{2}+a_{1} v+a_{0}}{\left(1+v^{2}\right)\left(v-v_{1}\right)\left(v-v_{2}\right)},
$$

where

$$
\begin{aligned}
& a_{3}=\frac{m(n+1)-k^{2}}{k^{2}-(m+1)(n+1)} \\
& a_{2}=\frac{m-(n+1) C_{H}}{k^{2}-(m+1)(n+1)} \\
& a_{1}=-\frac{n+1+C_{H}}{k^{2}-(m+1)(n+1)} \\
& a_{0}=-\frac{1}{k^{2}-(m+1)(n+1)},
\end{aligned}
$$

and $v_{1}$ and $v_{2}$ are the roots of the equation

$$
v^{2}-\frac{m+1-(n+1) C_{H}}{k^{2}-(m+1)(n+1)} v+\frac{C_{H}}{k^{2}-(m+1)(n+1)}=0 .
$$


Integral in $v$ can be written as

$\int \frac{d R}{R}=\int d v\left[\frac{A_{1}}{1+v^{2}}+\frac{2 A_{2} v}{1+v^{2}}+\frac{A_{3}}{v-v_{1}}+\frac{A_{4}}{v-v_{2}}\right]$.

Coefficients $A_{1}, A_{2}, A_{3}$ and $A_{4}$ are combinations of parameters from (53) (note that the minus sign in front of the integral is absorbed into these coefficients):

$$
\begin{aligned}
& A_{1}=\frac{\left(a_{0}-a_{2}\right)\left(1-v_{1} v_{2}\right)+\left(a_{1}-a_{3}\right)\left(v_{1}+v_{2}\right)}{\left(1+v_{1}^{2}\right)\left(1+v_{2}^{2}\right)} \\
& A_{2}=\frac{\left(a_{1}-a_{3}\right)\left(1-v_{1} v_{2}\right)-\left(a_{0}-a_{2}\right)\left(v_{1}+v_{2}\right)}{2\left(1+v_{1}^{2}\right)\left(1+v_{2}^{2}\right)} \\
& A_{3}=\frac{a_{3} v_{1}^{3}+a_{2} v_{1}^{2}+a_{1} v_{1}+a_{0}}{\left(1+v_{1}^{2}\right)\left(v_{2}-v_{1}\right)} \\
& A_{4}=-\frac{a_{3} v_{2}^{3}+a_{2} v_{2}^{2}+a_{1} v_{2}+a_{0}}{\left(1+v_{2}^{2}\right)\left(v_{2}-v_{1}\right)} .
\end{aligned}
$$

We can express $A$ 's directly trough $k, m, n$ and $C_{H}$, but it won't do us any good.

Integrating (59) gives us

$$
\begin{aligned}
\ln R= & A_{1} \arctan v+A_{2} \ln \left(1+v^{2}\right) \\
& +A_{3} \ln \left(v-v_{1}\right)+A_{4} \ln \left(v-v_{2}\right)+\ln C,
\end{aligned}
$$

where $C$ is integration constant.

Rewriting it a little, we obtain

$$
R=C\left(1+v^{2}\right)^{A_{2}}\left(v-v_{1}\right)^{A_{3}}\left(v-v_{2}\right)^{A_{4}} e^{A_{1} \arctan v} .
$$

Now, remember that $v=\cot \phi$. Then $\arctan v=$ $\arctan (\cot \phi)=\frac{\pi}{2}-\phi-\pi N$, where $N$ is an integer number. In our case $\phi \in[0,2 \pi]$, and we can absorb $A_{1} \frac{\pi}{2}$ into $C$. Finally, $1+v^{2}=1+\cot ^{2} \phi=\frac{1}{\sin ^{2} \phi}$, thus

$$
R=C \frac{\left(\cot \phi-v_{1}\right)^{A_{3}}\left(\cot \phi-v_{2}\right)^{A_{4}}}{\sin ^{A_{2}} \phi} e^{-A_{1} \phi} .
$$

The explicit expression for an integral curve depends on the number of the separatrices of the particular system. We integrated (53) assuming that the denominator has two real roots, which, according to (25) define separatrices. If the number of roots is different, as for the massless scalar field, for example, we have to integrate (53) again, and the expression for the integral curves will be different.

\section{CONCLUSIONS}

In this paper we have studied the Einstein equations in the case of the static cylindrical symmetry with the stress-energy tensor (1). Transformation (7)-(9) has allowed us to obtain system (10)-(11) that describes several physical cases, namely the cases of the static electric field, the perfect fluid and the cosmological constant. We have studied this system qualitatively, described its phase portraits in the whole phase plane. We have also obtained the expression for the integral curves of the system.

\section{ACKNOWLEDGMENTS}

The authors would like to express their gratitude to anonymous referees for providing valuable suggestions and remarks.
[1] S. Grigoryev, A. Leonov, Ukr. J. Phys. 58, 894 (2013).

[2] S. B. Grigoryev, in Proc. Int. Conf. GRG (GR-13), 1992.

[3] S. B. Grigoryev, Kinem. Fiz. Nebes. Tel 10, 25 (1994).

[4] N. N. Bautin, E. A. Leontovich, Metody i priemy kachestvennogo issledovanija dinamicheskikh sistem na ploskosti (Nauka, Moskva, 1990).

[5] A. A. Andronov et al., Kachestvennaja teorija dinamicheskikh sistem vtorogo porjadka (Nauka, Moskva, 1966).

[6] H. Stephani et al., Exact Solutions of Einstein's Field Equations, 2nd ed. (CUP, Cambridge, 2003).

[7] D. M. Chitre, R. Giiven, Y. Nutku, J. Math. Phys. 16, 475, (1975).

[8] N. Van den Bergh, P. Wils, J. Phys. A 16, 3843, (1983).

[9] M. A. H. MacCallum, J. Phys. A 16, 3853, (1983).

[10] H. Stephani et al., Exact Solutions of Einstein's Field Equations, 2nd ed. (CUP, Cambridge, 2003).

[11] A. K. Raychaudhuri, Ann. Phys. USA 11, 501 (1960).

[12] M. P. Korkina, S. B. Grigoryev, Ukr. Fiz. Zhurn. 29, 1153 (1984).

[13] A. B. Evans, J. Phys. A 10, 1303, (1977).
[14] T. G. Philbin, Clas. Quant. Grav. 13, 1217 (1996).

[15] S. Haggag, F. Desokey, Clas. Quant. Grav. 13, 3221 (1996).

[16] W. Davidson, Clas. Quant. Grav. 22, 553 (1990).

[17] U. S. Nilsson, C. Uggla, Clas. Quant. Grav. 14, 2931 (1997).

[18] T. Papacostas, J. Math. Phys. 29, 1445 (1988).

[19] K. A. Bronnikov, J. Phys. A 12, 201 (1979).

[20] A. Krasinski, Acta Phys. Polon. B 6, 223 (1975).

[21] C. Chicone, B. Mashhoon, Phys. Rev. D 83, 064013 (2011).

[22] A. A. Coley, preprint arXiv:gr-qc/9910074v1 (1974).

[23] A. A. Coley, Y. He, W. C. Lim , Clas. Quant. Grav. 21, 1311 (2004).

[24] M. A. Skugoreva, S. V. Sushkov, A. V. Toporensky, Phys. Rev. D 88, 083539 (2013).

[25] C. G. Boehmer, T. Harko, Clas. Quant. Grav. 24, 3191 (2007).

[26] N. Goheer, K. S. Peter, Phys. Rev. D 66, 043527 (2002).

[27] K. Yamamoto, Phys. Rev. D 85, 043510 (2012). 


\section{ЩОДО ЯКІСНОГО ДОСЛІДЖЕННЯ ДЕЯКИХ СТАТИЧНИХ ЦИЛІНДРИЧНО-СИМЕТРИЧНИХ РОЗВ'ЯЗКІВ РІВНЯНЬ АЙНШТАЙНА}

С. Б. Григор'єв, А. Б. Леонов

Дніпропетровсъкий начіональний університет ім. Олеся Гончара, просп. Гагаріна, 72, Дніпропетровсък, 49010, Украӥна

Ми проводимо якісне дослідження рівнянь Айнштайна у статичній циліндричній симетрії з діагональним тензором енергії-імпульсу зі взаємно пропорційними компонентами. Цей випадок охоплює декілька важливих точних розв'язків рівнянь Айнштайна, а саме, розв'язок зі статичним електричним полем, ідеальна рідина, розв'язок із космологічною сталою та випадок безмасового скалярного поля. Система рівнянь має складну точку рівноваги, але завдяки особливостям самої системи можна провести її якісне дослідження в усій фазовій площині. 